\title{
Autophagy, a new target for disease treatment
}

\author{
XIE Feng, LI LanFang \& CHEN LinXi* \\ Institute of Pharmacy and Pharmacology, University of South China, Hengyang 421001, China
}

Received May 20, 2013; accepted June 17, 2013; published online August 7, 2013

Citation: Xie F, Li L F, Chen L X. Autophagy, a new target for disease treatment. Sci China Life Sci, 2013, 56: 856-860, doi: 10.1007/s11427-013-4530-0

Since Ashford and Porter first discovered that cells "eat themselves" in 1962, the process known as autophagy has generated intense interest in both biomedical research and clinical medicine. Autophagy is a regulated cellular pathway that transports damaged, modified or aging organelles and proteins to lysosomes/vacuoles for degradation. But recently, autophagy has been identified as being not only a widespread normal physiological process, but also a cell defense mechanism in response to an adverse cellular environment, directly related to the pathological processes of clinical diseases, including cardiovascular disease, lung disease, cancer, neurodegenerative disease and infection. The role of autophagy in diseases can be either beneficial or harmful: normal levels of autophagy can protect cells from environmental stimuli; however, continued excessive or insufficient autophagy can lead to disease. The level of autophagy may be reflective of the development of diseases and may provide a target for disease treatment.

Autophagy is a lysosome-mediated, bulk degradative process by which eukaryotes recycle organelles and longduration proteins. Based on the distinctive degradative mechanisms, autophagy is conventionally described in three main forms: (i) chaperone-mediated autophagy; (ii) microautophagy; and (iii) macroautophagy [1]. Macroautophagy involves the formation of a dynamic subcellular organelle, a so-called "autophagosome". Autophagosomes play a very important role in cell degradation and have a close relationship with diseases of protein transport dysfunction and degradation. A multivesicular body is another dynamic subcellular organelle formed by invagination of the limited mem-

*Corresponding author (email: chenlinxi@tom.com) brane of a late endosome and is involved in various processes including protein transport and sorting. Pashkova et al. [2] have discussed the assembly mechanisms, interactions and functions of ESCRT complexes (the endosomal sorting complex required for transport) by ubiquitinated proteins and ubiquitination in multivesicular bodies. This research provides new information about protein transport and degradation in cells, and may provide links to some diseases in terms of abnormalities of protein degradation.

The process of autophagy is well-regulated, and the protein kinase target of rapamycin, TOR, is a key inhibitory regulator. Under starvation conditions, or in the presence of rapamycin, TOR kinase is inactive and autophagic activity is enhanced. In response to insulin-like and other growth factor signals, the class I PI3K/Akt signaling molecules link receptor tyrosine kinases to activate TOR kinase and thereby repress autophagy [3]. Class III phosphoinositide 3-kinase (class III PI3K), which specifically phosphorylates PtdIns to generate PtdIns3P, combined with beclin1 to form a class III PI3K-Beclin1 complexes, also plays a vital role in autophagy and vacuolar sorting pathways [4].

Autophagy is not only a widespread normal physiological process, but is also an important mechanism that assists cells in adapting to environmental change, provides a defense against the invasion of pathogenic microorganisms and aids in the maintenance of homeostasis. Recently, it has been discovered that autophagy not only has an effect on cellular homeostasis but also has an effect on organs and is involved in the pathological processes of clinical disease. By having a function in disease, autophagy has become a target in some disease treatments, particularly in some cardiovascular, neuronal, lung and metabolism diseases and in 
tumor development and infection.

\section{Autophagy in cardiovascular disease}

Despite the ongoing advances in treatment options, cardiovascular disease remains a leading cause of death in China and is reaching pandemic scales worldwide. The dysfunction and loss of myocytes is key in the pathology of cardiovascular disease, particularly in terms of the cellular autophagic pathway. It is well known that mitochondrial balance can maintain the function of myocytes. Therefore, selective autophagy of mitochondria, termed "mitophagy", plays a very important role in maintaining myocyte function [5]. Lizama-Manibusan et al. [6] has summarized that redox-sensitive proteins regulate mitophagy and ultimately promote cell survival in diseases including Parkinson's, Amyotrophic Lateral Sclerosis, and cancer.

At present, the exact role of autophagy in the heart is highly debated, but it has been suggested to play a key role in regulating cell turnover in cardiomyopathies and heart failure. Xie et al. [7] surmised that dysfunctional myocardial autophagy contributes to a diverse set of heart diseases, such as Danon disease, myocardial hypertrophy and heart failure. Vascular endothelial cells (VEC) are one of the most active cells in the human body, and many cardiovascular diseases are correlated with functional changes to VEC. For this reason, VEC autophagy is another target in the treatment of cardiovascular diseases, especially atherosclerosis. Epigallocatechin-gallate (EGCG) stimulates autophagy in vascular endothelial cells and become a potential therapeutic reagent to prevent cardiovascular complications [8]. These reports suggest that autophagy in myocytes and VEC are crucial factors in cardiovascular disease.

\section{Autophagy in neurons}

In neurons, autophagy has been reported as being active in the acute phase after subarachnoid hemorrhage. The activation of autophagy decreased neuronal apoptosis and ameliorated early brain injury EBI after subarachnoid hemorrhage and may, therefore, be a potential effective target for preventing EBI after subarachnoid hemorrhage [9]. Endoplasmic reticulum (ER) stress-induced autophagy might contribute to the neuroprotective effect of brain ischemic preconditioning [10]. However, it has also been reported that hypertension can induce excessive autophagic activation in the brain, leading to cell death, and subsequently induce several disorders. Angiotensin-(1-7) exerts neuroprotective effects by preventing hypertension-induced excessive autophagic activation, reducing oxidative stress and apoptosis in the brains of spontaneously hypertensive rats [11]. Autophagy vacuoles were also frequently observed in neurodegenerative diseases such as Alzheimer's, Parkin- son's, and Huntington's diseases as well as prion diseases. A large amount of data show that autophagy has a protective role in neurodegeneration: autophagy deficiency is a secondary disease mechanism in neurodegeneration and induction of autophagy can act therapeutically in neurodegenerative disorders [12]. Prion diseases, characterized by spongiform degeneration and the accumulation of misfolded and aggregated PrPSc in the central nervous system, are among the fatal neurodegenerative and infectious disorders in humans and animals. Autophagy mediates neuronal toxicity or serves a protective function in prion diseases, and some small molecules (such as lithium, rapamycin, Sirtuin 1 and resveratrol) mitigate such diseases of brain function [13]. The above research suggests that autophagy can protect from neurodegeneration. The regulation of autophagy in neurons may counter the development of neurodegenerative diseases.

\section{Autophagy in cancer}

Autophagy is an evolutionarily conserved catabolic process crucial for cell death and survival, and also provides an important function in tumor development. Changes in macroautophagic activity have been described in cancer cells and in solid tumors, and inhibition of macroautophagy promotes tumorigenesis. Autophagy is emerging as an important biological mechanism in targeting human cancers, including cervical cancer [14], liver cancer [15], colorectal cancer [16], colon cancer [17], and prostate cancer [18] and autophagy suppresses tumor progression by limiting genome instability, promotes tumor cell survival and restricts necrosis, inflammation, and tumorigenesis.

\section{Autophagy in lung disease}

Autophagy is closely related to the occurrence and development of lung diseases, where its activity can fluctuate. Liu et al. [19] reported that the IL17A-PI3K-GSK3 $\beta-B C L 2$ signaling pathway participates in the attenuation of autophagic activity in lung epithelial cells, which is considered to be primarily responsible for the development and progression of IL17A-induced pulmonary fibrosis. The downregulation of autophagy may lead to systemic inflammation and acute lung injury following sepsis. The direct or indirect modification of autophagy using rapamycin resulted in improved survival. Enhancing or restoring autophagy early after sepsis seems to be a potential strategy for the treatment of sepsis-induced acute lung injury [20]. Yang et al. [21] draw the conclusion that there is a relationship between autophagy and lung diseases, including pulmonary emphysema, chronic obstructive pulmonary disease, lung cancer and pulmonary tuberculosis. This research helps in understanding the autophagic process, the regulation of au- 
tophagy in lung diseases, and provides new ideas for therapies for lung diseases.

\section{Autophagy and pathogens}

Autophagy is associated with the formation of autophagosomes, and further functions as a defense mechanism against infection by various pathogens. Lupfer et al. [22] reported that nucleotide-binding oligomerization domain 2 receptor and receptor interacting protein kinase 2 signaling can protect against virally triggered immunopathology by negatively regulating activation of NOD like receptors family, pyrin domain containing three inflammasomes and production of IL-18 via Unc-51-like kinase 1-dependent mitophagy. Some pathogens, such as Salmonella enterica serovar Typhimurium and Mycobacterium tuberculosis, succumb to autophagy and are transported to lysosomes for degradation, yet many pathogens, including human papillomavirus (HPV)16 and oncolytic viruses, subvert this pathway, exploiting autophagy to their advantage. For example, Ishii [23] found that autophagy is indeed induced during the HPV16 entry process, and oncolytic viruses perturb cellular autophagy machinery in infected tumor cells both in vitro and in vivo [24]. During Mycobacterium tuberculosis infection, autophagy acts not only as an antimicrobial mechanism for the clearance of intracellular pathogen, but also prevents excessive inflammation, preventing adverse effects on the host. Novel autophagy-based therapy is being considered as a better tuberculosis medication [25]. HIV protease inhibitors (PI) are core components of highly active anti-retroviral therapy, the most effective treatment for HIV infection currently available. However, HIV PIs have now been linked to lipodystrophy and dyslipidemia, which are major risk factors for cardiovascular disease and metabolic syndrome. Zha et al. [26] found that activation of ER stress and impairment of autophagy activity are involved in HIV PI-induced dysregulation of lipid metabolism in adipocytes. The key components of ER stress and autophagy signaling pathways are potential therapeutic targets for the HIV PI-induced metabolic side effects in HIV patients. Historically, influenza pandemics have arisen from avian influenza viruses. Avian influenza viruses $\mathrm{H} 5 \mathrm{~N} 1$ and H9N2 are potential pandemic candidates. It is reported that autophagic responses play a role in influenza virus-induced CXCL10 and interferon- $\alpha$ expression in primary human blood macrophages [27]. Accumulation of autophagosomes has also been observed in H5N1-infected lungs from a human cadaver, from mice, as well as in infected A549 human epithelial lung cells. H5N1 caused autophagic cell death through suppression of mTOR signaling [28]. Autophagic cell death of alveolar epithelial cells plays a crucial role in the high mortality rates of $\mathrm{H} 5 \mathrm{~N} 1$ infection. Autophagy-blocking agents might be useful as prophylactics and therapeutics against human infection by the H5N1 virus
[29]. An anti-influenza agent, human monoclonal ScFv, has anti-autophagic function [30]. Recently, a new avian influenza virus, H7N9, emerged as a serious threat to humans. Further research into the role of autophagy in avian influenza virus infection may provide new therapies to combat infection.

\section{Autophagy and metabolism}

Autophagy is a process for the degradation of proteins and organs. It therefore plays a role in the regulation of metabolism in cells. Autophagy and lipid homeostasis (both ancient cellular pathways) have seemingly co-evolved to share common regulatory elements, and autophagy has emerged as a prominent mechanism involved in the regulation of lipid metabolism. Autophagy is involved in some diseases characterized by a dysregulation of lipid metabolism, such as obesity, diabetes and atherosclerosis [31]. Autophagy is required for adipocyte differentiation and the concurrent accumulation of lipid droplets, and it also affects lipid metabolism through contributions to lipoprotein [32]. It is reported that ketogenic amino acid replacement in food could be a novel strategy to combat hepatic steatosis and metabolic abnormalities, possibly through induction of autophagy [33]. It is also reported that obesity and excessive calorie intake are associated with the defective regulation of autophagy in adipose tissue [34], and insulin resistance in adipose tissue of obese Ristar Ottawa Karlsburg W (RT1u) rats is associated with upregulation of differing autophagic markers in visceral and subcutaneous fat deposits [35]. MXL-3 and HLH-30 act as transcriptional switches coupling lysosomal lipolysis and autophagy to nutrient availability and controlling fat storage and ageing in Caenorhabditis elegans [36]. In obesity, autophagy helps maintain $\beta$-cell mass, structure and function; autophagy-deficient $\beta$-cells can handle basal metabolic stress but have problems dealing with increased metabolic stress [37]. These studies suggest that autophagy is a vital regulator of metabolic disease.

\section{Levels of autophagy for disease detection}

Normal levels of autophagy can protect cells from environmental stimuli; however, continued excessive or insufficient autophagy can lead to disease. The level of autophagy may reflect the degree of some diseases, therefore detection of the level of autophagy may be an indirect way to evaluate some diseases. Dysfunction of autophagy involves formation and degeneration of autophagosomes or the overexpression of some autophagy-related proteins. Accordingly, accurate and comprehensive evaluation of autophagy includes autophagosome detection, as well as the fluency of autophagic degeneration, for example, autophagic flux as- 
say. At present, transmission electron microscopy [38,39], immunofluorescence and immunoblotting techniques are commonly used to detect autophagy. However, these methods cannot describe the dynamic process of autophagy. More accurate detection methods are needed. Additionally, artificial up- or downregulation of autophagy by drugs (for example, autophagy inducers like rapamycin and inhibitors like 3-MA) or gene interferences in vitro (for example, RNA interfere Atg3, Atg5, Atg7 and Beclin1) and in vivo (for example, GFP-LC3 transgenic mice [40,41]) have also been considered as an important part of autophagy analysis. Any method used alone may not provide sufficient evidence of autophagy. Careful attention needs to be paid to the results of any assays for autophagy [42].

\section{Conclusion}

With its roles in cardiovascular disease, lung disease, cancer and viral infection, autophagy's importance in disease initiation and progression is just beginning to be understood. More researchers are turning their attention to the role of autophagy in disease treatment. Zou et al. [43] reported that colostrum contains a high level of miR-30a, and this miR-30a restrained autophagy activity while promoting apoptosis of HeLa cells induced by cis-DDP (cis-dichloride diamine platinum, cis-DDP, $\mathrm{Pt}(\mathrm{NH} 3) 2 \mathrm{Cl} 2$ ). The miR-30arich fraction of colostrum also reduced tumor size more effectively than did treatment with DDP alone in mice. It is suggested that the miR-30a-rich fraction of colostrum can sensitize cancer cells to anti-cancer drugs, thus enhancing the effect of chemotherapeutic drugs during tumor treatment. Chen et al. [44] reported that miR-221/222 acts as a promising biomarker for breast cancer by regulating autophagy, and that it may offer a new way for molecular targeting in cancer treatment. These reports suggest that autophagy may become a target for cancer therapy in the future. It has been reported that rapamycin, an inducer of autophagy, can inhibit certain tumor cell growths, including rhabdomyosarcoma, neuroblastoma, small-cell lung carcinoma, osteosarcoma, pancreatic carcinoma, renal cell carcinoma, prostate cancer and breast cancer. Some rapamycin analogs, such as CCI-779/temsirolimus [45] and RAD-001/everoli- mus [46], have been used to treat tumors such as glioblastoma, prostate cancer and breast cancer. Recent studies with omeprazole, which is commonly used in gastritis treatment, have found that it inhibits the growth of tumor cells by inducing autophagy and enhances the function of 5-fluor- ouracile [47]. Chloroquine, an inhibitor of autophagy, can enhance the function of imatinibmesylate in Philadelphia chromosome-positive cells and chronic myeloid leukemia treatment. Another autophagy inhibitor, bafilomycinA1, has the same role in chronic myeloid leukemia [48]. Xie et al. [7] have reported that some anti-tumor drugs (for example, doxorubicine, daunorubicin, resveratrol, temozolomide, $\mathrm{As}_{2} \mathrm{O}_{3}$, gemcitabine and paclitaxel) and some cardiovascular regulators (for example, angiotensin II, carvedilol and atorvastatin) can induce autophagy, but it is not clear how best to regulate autophagy using these drugs or whether autophagy is indeed an appropriate target for these drugs in the treatment of disease.

It is becoming apparent that autophagy is not only a physiological phenomenon for cells, but could possibly become a mechanism for the development of approaches in the treatment of disease. More biomedical scientists are turning their attention to this remarkable cellular process. Because autophagy is a dynamic and complex process, and the regulation of autophagy is complex, the level of autophagy at a particular time and in a particular situation is very hard to detect. The role of autophagy in disease can be either beneficial or harmful. A more complete characterization of the autophagic machinery needs to be described. Alongside gaining an understanding of the implications of autophagy in disease, future research will need to focus on how to use autophagy as a target in the treatment of diseases.

This work was supported by the National Natural Science Foundation of China (30901577, 81270420), Hengyang Joint Funds of Hunan Provincial Natural Science Foundation of China (12JJ8013).

1 Mizushima N, Komatsu M. Autophagy: renovation of cells and tissues. Cell, 2011, 147: 728-741

2 Pashkova N, Gakhar L, Winistorfer S C, et al. The yeast alix homolog bro1 functions as a ubiquitin receptor for protein sorting into multivesicular endosomes. Dev Cell, 2013, 25: 520-533

3 Shinji S, Yukiko S, Yoko I, et al. Caffeine induces apoptosis by enhancement of autophagy via PI3K/Akt/mTOR/p70S6K inhibition. Autophagy, 2011, 7: 176-187

4 Amina T, Marie Paule R, Didier A, et al. Class III phosphoinositide 3-kinase-Beclin1 complex mediates the amino acid-dependent regulation of autophagy in $\mathrm{C}_{2} \mathrm{C}_{12}$ myotubes. Biochem J, 2003, 376: 577-586

5 Lemasters J J. Selective mitochondrial autophagy, or mitophagy, as a targeted defense against oxidative stress, mitochondrial dysfunction, and aging. Rejuv Res, 2005, 8: 3-5

6 Lizama-Manibusan B, McLaughlin B. Redox modification of proteins as essential mediators of CNS autophagy and mitophagy. FEBS Lett, 2013, 587: 2291-2298

7 Xie F, Liu W, Chen L X. The Progress of autophagy involved in heart disease. Prog Biochem Biophys, 2012, 39: 224-233

8 Kim H S, Montana V, Jang H J, et al. Epigallocatechin-gallate (EGCG) stimulates autophagy in vascular endothelial cells: a potential role for reducing lipid accumulation. J Biol Chem, 2013, doi: 10.1074/jbc.M113.477505

9 Zhao H, Ji Z, Tang D, et al. Role of autophagy in early brain injury after subarachnoid hemorrhage in rats. Mol Biol Rep, 2013, 40: 819-827

10 Gao B, Zhang X Y, Han R, et al. The endoplasmic reticulum stress inhibitor salubrinal inhibits the activation of autophagy and neuroprotection induced by brain ischemic preconditioning. Acta Pharmacol Sin, 2013, 34: 657-666

11 Jiang T, Gao L, Zhu X C, et al. Angiotensin-(1-7) inhibits autophagy in the brain of spontaneously hypertensive rats. Pharmacol Res, 2013, 71: 61-68

12 Winslow A R, Rubinsztein D C. Autophagy in neurodegeneration and development. Biochim Biophys Acta, 2008, 1782:723-729 
13 Yao H, Zhao D, Khan S H, et al. Role of autophagy in prion protein-induced neurodegenerative diseases. Acta Biochim Biophys Sin (Shanghai), 2013, 45: 494-502

14 Pandey S, Chandravati. Autophagy in cervical cancer: an emerging therapeutic target. Asian Pac J Cancer Prev, 2012, 13: 4867-4871

15 Cui J, Gong Z, Shen H M. The role of autophagy in liver cancer: molecular mechanisms and potential therapeutic targets. Biochim Biophys Acta, 2013, 1836: 15-26

16 Xie X L, Liu Y B, Liu Y P, et al. Reduced expression of SM22 is correlated with low autophagy activity in human colorectal cancer. Pathol Res Pract, 2013, 209: 237-243

17 Kwatra D, Subramaniam D, Ramamoorthy P, et al. Methanolic extracts of bitter melon inhibit colon cancer stem cells by affecting energy homeostasis and autophagy. Evid Based Complement Alternat Med, 2013, 2013: 702869

18 Boutin B, Tajeddine N, Vandersmissen $\mathrm{P}$, et al. Androgen deprivation and androgen receptor competition by bicalutamide induce autophagy of hormone-resistant prostate cancer cells and confer resistance to apoptosis. Prostate, 2013, 73: 1090-1102

19 Liu H, Mi S, Li Z, et al. Interleukin 17A inhibits autophagy through activation of PIK3CA to interrupt the GSK3B-mediated degradation of BCL2 in lung epithelial cells. Autophagy, 2013, 9: 730-742

20 Yen Y T, Yang H R, Lo H C, et al. Enhancing autophagy with activated protein $\mathrm{C}$ and rapamycin protects against sepsis-induced acute lung injury. Surgery, 2013, 153: 689-698

21 Yang L, Xiao L, Chen L X. Research progress of autophagy and pulmonary diseases. Prog Biochem Biophys, 2012, 39: 861-868

22 Lupfer C, Thomas P G, Anand P K, et al. Receptor interacting protein kinase 2-mediated mitophagy regulates inflammasome activation during virus infection. Nat Immunol, 2013, 14: 480-488

23 Ishii Y. Electron microscopic visualization of autophagosomes induced by infection of human papillomavirus pseudovirions. Biochem Biophys Res Commun, 2013, 433: 385-389

24 Meng S, Xu J, Wu Y, et al. Targeting autophagy to enhance oncolytic virus-based cancer therapy. Expert Opin Biol Ther, 2013, 13: 863-873

25 Yu X, Li C, Hong W, et al. Autophagy during Mycobacterium tuberculosis infection and implications for future tuberculosis medications. Cell Signal, 2013, 25: 1272-1278

26 Zha B S, Wan X, Zhang X, et al. HIV protease inhibitors disrupt lipid metabolism by activating endoplasmic reticulum stress and inhibiting autophagy activity in adipocytes. PLoS ONE, 2013, 8: e59514

27 Law A H, Lee D C, Yuen K Y, et al. Cellular response to influenza virus infection: a potential role for autophagy in CXCL10 and interferon-alpha induction. Cell Mol Immunol, 2010, 7: 263-270

28 Sun Y, Li C, Shu Y, et al. Inhibition of autophagy ameliorates acute lung injury caused by avian influenza A H5N1 infection. Sci Signal, 2012, 5: ra16

29 Ma J, Sun Q, Mi R, et al. Avian influenza A virus H5N1 causes autophagy-mediated cell death through suppression of mTOR signaling. J Genet Genomics, 2011, 38: 533-537

30 Pissawong T, Maneewatch S, Thueng-In K, et al. Human monoclonal $\mathrm{ScFv}$ that bind to different functional domains of M2 and inhibit H5N1 influenza virus replication. Virol J, 2013, 10: 148

31 Ouimet M. Autophagy in obesity and atherosclerosis: interrelation- ships between cholesterol homeostasis, lipoprotein metabolism and autophagy in macrophages and other systems. Biochim Biophys Acta, 2013, 1831: 1124-1133

32 Christian P, Sacco J, Adeli K. Autophagy: emerging roles in lipid homeostasis and metabolic control. Biochim Biophys Acta, 2013, 1831: 819-824

33 Xu L, Kanasaki M, He J, et al. Ketogenic essential amino acids replacement diet ameliorated hepatosteatosis with altering autophagy-associated molecules. Biochim Biophys Acta, 2013, 1832: 1605-1612

34 Nunez C E, Rodrigues V S, Gomes F S, et al. Defective regulation of adipose tissue autophagy in obesity. Int $\mathrm{J}$ Obes (Lond), 2013, doi: 10.1038/ijo.2013.27

35 Kosacka J, Koch K, Gericke M, et al. The polygenetically inherited metabolic syndrome of male WOKW rats is associated with enhanced autophagy in adipose tissue. Diabetol Metab Syndr, 2013, 5: 23

36 O'Rourke E J, Ruvkun G. MXL-3 and HLH-30 transcriptionally link lipolysis and autophagy to nutrient availability. Nat Cell Biol, 2013, 15: 668-676

37 Quan W, Jung H S, Lee M S. Role of autophagy in the progression from obesity to diabetes and in the control of energy balance. Arch Pharm Res, 2013, 36: 223-229

38 Swanlund J M, Kregel K C, Oberley T D. Investigating autophagy: quantitative morphometric analysis using electron microscopy. Autophagy, 2010, 6: 270-277

39 Yla-Anttila P, Vihinen H, Jokitalo E, et al. Monitoring autophagy by electron microscopy in mammalian cells. Methods Enzymol, 2009, 452: $143-164$

40 Mizushima N. Methods for monitoring autophagy using GFP-LC3 transgenic mice. Methods Enzymol, 2009, 452: 13-23

41 Tian F, Deguchi K, Yamashita T, et al. In vivo imaging of autophagy in a mouse stroke model. Autophagy, 2010, 6: 1107-1114

42 Ma T, Sun G P, Li J B. Methods for autophagy detection. Prog Biochem Biophys, 2012, 39: 204-209

43 Zou Z Y, Wang Y, Ding $\mathrm{H}$, et al. miR-30a Enriched colostrum-fraction can inhibit the ddp-induced autophagy of HeLa cells and promote the tumor shrink. Prog Biochem Biophys, 2012, 39: 1099-1108

44 Chen W X, Hu Q, Qiu M T, et al. miR-221/222: promising biomarkers for breast cancer. Tumour Biol, 2013, 34: 1361-1370

45 Atkins M B, Hidalgo M, Stadler W M, et al. Randomized phase II study of multiple dose levels of CCI-779, a novel mammalian target of rapamycin kinase inhibitor, in patients with advanced refractory renal cell carcinoma. J Clin Oncol, 2004, 22: 909-918

46 Cao C, Subhawong T, Albert J M, et al. Inhibition of mammalian target of rapamycin or apoptotic pathway induces autophagy and radiosensitizes PTEN null prostate cancer cells. Cancer Res, 2006, 66: 10040-10047

47 Udelnow A, Kreyes A, Ellinger S, et al. Omeprazole inhibits proliferation and modulates autophagy in pancreatic cancer cells. PLoS ONE, 2011, 6: e20143

48 Bellodi C, Lidonnici M R, Hamilton A, et al. Targeting autophagy potentiates tyrosine kinase inhibitor-induced cell death in Philadelphia chromosome-positive cells, including primary CML stem cells. J Clin Invest, 2009, 119: 1109-1123

Open Access This article is distributed under the terms of the Creative Commons Attribution License which permits any use, distribution, and reproduction in any medium, provided the original author(s) and source are credited. 